\title{
Reliability of Bioelectrical Impedance Analysis for Estimating Whole-Fish Energy Density and Percent Lipids
}

\author{
Steven A. Pothoven* \\ National Oceanic and Atmospheric Administration, Great Lakes Environmental Research Laboratory, \\ 1431 Beach Street, Muskegon, Michigan 49441, USA \\ STUART A. Ludsin ${ }^{1}$ \\ National Oceanic and Atmospheric Administration, Great Lakes Environmental Research Laboratory, \\ 2205 Commonwealth Boulevard, Ann Arbor, Michigan 48105 USA
}

TOMAS O. HöÖK ${ }^{2}$

Cooperative Institute of Limnology and Ecosystem Research, University of Michigan, and National Oceanic and Atmospheric Administration, Great Lakes Environmental Research Laboratory, 2205 Commonwealth Boulevard, Ann Arbor, Michigan 48105 USA

\section{David L. Fanslow and Doran M. Mason}

National Oceanic and Atmospheric Administration, Great Lakes Environmental Research Laboratory, 2205 Commonwealth Boulevard, Ann Arbor, Michigan 48105, USA

\section{Paris D. Collingsworth and Jason J. Van Tassell}

Aquatic Ecology Laboratory, Department of Evolution, Ecology and Organismal Biology, Ohio State University, 1314 Kinnear Road, Columbus, Ohio 43212, USA

\begin{abstract}
We evaluated bioelectrical impedance analysis (BIA) as a nonlethal means of predicting energy density and percent lipids for three fish species: yellow perch Perca flavescens, walleye Sander vitreus, and lake whitefish Coregonus clupeaformis. Although models that combined BIA measures with fish wet mass provided strong predictions of total energy, total lipids, and total dry mass for whole fish, including BIA provided only slightly better predictions than using fish mass alone. Regression models that used BIA measures to directly predict the energy density or percent lipids of whole fish were generally better than those using body mass alone (based on Akaike's information criterion). However, the goodness of fit of models that used BIA measures varied widely across species and at best explained only slightly more than one-half the variation observed in fish energy density or percent lipids. Models that combined BIA measures with body mass for prediction had the strongest correlations between predicted and observed energy density or percent lipids for a validation group of fish, but there were significant biases in these predictions. For example, the models underestimated energy density and percent lipids for lipid-rich fish and overestimated energy density and percent lipids for lipid-poor fish. A comparison of observed versus predicted whole-fish energy densities and percent lipids demonstrated that models that incorporated BIA measures had lower maximum percent error than models without BIA measures in them, although the errors for the BIA models were still generally high (energy density: 15-18\%; percent lipids: $82-89 \%$ ). Considerable work is still required before BIA can provide reliable predictions of whole-fish energy density and percent lipids, including understanding how temperature, electrode placement, and the variation in lipid distribution within a fish affect BIA measures.
\end{abstract}

The ability to quantify the energy content of fish has benefited both fisheries management and ecological

\footnotetext{
* Corresponding author: steve.pothoven@noaa.gov

${ }^{1}$ Present address: Aquatic Ecology Laboratory, Department. of Evolution, Ecology, and Organismal Biology, Ohio State University, 1314 Kinnear Road, Columbus, Ohio 43212, USA.

${ }^{2}$ Present address: Department of Forestry and Natural Resources, Purdue University, 195 Marsteller Street, West Lafayette, Indiana 47907-2033, USA.
}

Received August 31, 2007; accepted March 7, 2008 Published online September 22, 2008 investigations (Rottiers and Tucker 1982; Henderson et al. 1996; Hurst and Conover 2003; Madenjian et al. 2006). Such information can be used to describe the flow of energy between and within populations and evaluate an individual's or population's response to ecosystem changes (Paine 1971; Ludsin and DeVries 1997; Madenjian et al. 2000). Similarly, measures of energy content such as energy density and percent lipids can provide insight into the physiological status of a fish, which may reflect its condition for wintering, migrating, or spawning (Rottiers and Tucker 1982; Shearer 1994; Ludsin and DeVries 1997). Insufficient 
information on fish energy content-specifically, energy density - can also compromise bioenergetic and energy flow studies (Bartell et al. 1986; Vondracek et al. 1996; Lantry et al. 1999).

Despite the potential value of quantifying energy content, there is surprisingly little information for most fish species and even less on how measures of energy content (e.g., energy density, percent lipids) vary ontogenetically, seasonally, and geographically. In part, this information gap is undoubtedly due to the expense and time required to quantify energy content with traditional laboratory approaches (Vondracek et al. 1996; Lantry et al. 1999). Further, because traditional methods used to assess fish energy content require individuals to be sacrificed, such approaches cannot be used to quantify energy content on the same individual through time and are not appropriate for species that are low in abundance, threatened, or endangered.

Alternative approaches have been developed to estimate the energy content of fish. For example, some studies have demonstrated that energy density and lipid content can vary with fish size; however, the amount of variation attributable to fish size can differ among species, and in some cases, the relationship changes as fish grow (Shearer 1994; Madenjian et al. 2000; Pothoven et al. 2006). Likewise, Hartman and Brandt (1995) found that ratio of dry to wet mass of an individual can predict the energy density of fish quite well. However, this method still necessitates sacrificing fish and requires substantial time for grinding and drying fish carcasses. Clearly, development of a rapid, nonlethal approach for estimating proximate body composition-including energy density and percent lipids - would be of great value to fishery scientists and management agencies.

Several technological developments have provided an alternative to the time-consuming determination of energy content in fish, including total-body electrical conductivity (TOBEC), handheld microwave energy meters (fat meters), and bioelectrical impedance analysis (BIA). Both TOBEC and BIA rely on the differing conductive properties of fat and fat-free mass within a body to evaluate whole-body lipid content, whereas microwave energy meters use a microwave sensor to measure water content in a cell and then use species-specific relationships to determine somatic lipid content (Crossin and Hinch 2005). The TOBEC instruments are large and difficult to use in field situations and have not proven reliable for fisheries studies (Lantry et al. 1999). In contrast, both BIA and microwave energy instruments are more portable. Given our interest in estimating whole-body energy content (not just somatic tissue content) in field settings in a nonlethal manner, we chose to evaluate the use of BIA for this study.

The theory of BIA for estimating total-body water in humans was established by 1970 and increasingly has been used for clinical applications (Kushner et al. 1992; Kotler et al. 1996; Kyle et al. 2004). More recently, BIA has shown some potential for estimating body composition of other animals, including moose Alces alces (Hundertmark and Schwartz 2002), gray seals Halichoerus grypus (Bowen et al. 1999), and more recently, fish (Bosworth and Wolters 2001; Cox and Hartman 2005). BIA is based on the principle that impedance in a simple geometric system is a function of conductor length, its cross-sectional area, and the applied signal frequency (Hoffer et al. 1969; Lukaski et al. 1985; Kushner et al. 1992; Kyle et al. 2004). The theory underlying BIA assumes that water offers less resistance to electrical current than lipids, that is, that higher resistance equates to higher amounts of lipids or nonconductive materials such as bone (Lukaski et al. 1985; Jackson et al. 1988; Kyle et al. 2004). Reactance provides a measure of the volume of cell membrane capacitance and in theory is not affected by the fat quantity in a body (Lukaski et al. 1985). BIA measures can be correlated with measures of proximate body composition to develop calibration equations to predict proximate body composition (Kushner et al. 1992; Kyle et al. 2004; Cox and Hartman 2005).

Previous work on whole fish has used BIA to successfully predict total-body water and lipids $\left(R^{2}>\right.$ 0.96; Cox and Hartman 2005) but not caloric energy density, the metric most commonly used to evaluate fish health and track energy flow with bioenergetics models (Rottiers and Tucker 1982; Hanson et al. 1997; Lantry et al. 1999). Considering the strong correlation between water content and energy density (Hartman and Brandt 1995), we sought to determine whether BIA could provide robust predictions of whole-fish energy density for yellow perch Perca flavescens, walleye Sander vitreus, and lake whitefish Coregonus clupeaformis. These fish are of recreational, commercial, or ecological importance in the Great Lakes and differ considerably in terms of body size and whole-body energy content. Additionally, because most studies evaluating BIA have examined only the relationship between BIA and total lipid mass, we wanted to determine whether BIA could predict percent lipids, another metric commonly used to evaluate fish condition (Madenjian et al. 2000; Hendry and Beall 2004). If BIA can provide a rapid and nonlethal manner to determine whole-fish energy content, then fishery managers and scientists would have a nonlethal tool to quickly and easily track the physiological status of fish in the field. Bioenergetics applications could thus be 
improved through the collection of more seasonal and interannual species-specific data on fish energy content.

\section{Methods}

Yellow perch, walleyes, and lake whitefish were collected with either gill nets or bottom trawls from (1) Lake Erie during May and September 2005 (yellow perch) and (2) Lake Michigan and a connected drowned river mouth (Muskegon Lake) during JuneOctober 2006 (walleyes, yellow perch, and lake whitefish). An additional sample of walleyes (originating from Lake Erie) was taken from Michigan Department of Natural Resources experimental ponds during April 2005. When collected, fish were measured (total length [TL], nearest $\mathrm{mm}$ ) and weighed (nearest g). We anticipated that we would collect a broad range of fish sizes and fish with various energy contents by sampling various systems during different seasons. While fish were still alive, they were placed on a nonconductive board and bioelectrical impedance (resistance and reactance, series) was quantified by applying an $800 \mu \mathrm{A}$ current at $50 \mathrm{kHz}$ with a Quantum II bioelectrical impedance analyzer (RJL Systems, Detroit, Michigan). After BIA measures were taken, the fish were euthanized, individually wrapped in aluminum foil to reduce moisture loss, bagged, and frozen.

The BIA system consisted of two sets of 3-gauge, 19mm-long hypodermic needles. Each set included an outer transmitting and an inner detecting electrode held $1 \mathrm{~cm}$ apart in a plastic housing that allowed each needle to penetrate about $3 \mathrm{~mm}$ into the fish muscle. For the lateral placement of electrodes, one transmitting electrode was placed just above the anterior end of operculum so that its paired detecting electrode was 1 $\mathrm{cm}$ posterior to the operculum. The other pair of electrodes was placed so that the detecting electrode was even with the posterior edge of the second dorsal fin (for walleyes and yellow perch) or the transmitting electrode was even with the anterior edge of the adipose fin (for lake whitefish; Cox and Hartman 2005). A current was then introduced by the outer transmitting electrodes and the voltage drop between the two inner detecting electrodes was determined. Dorsal BIA measures were also taken (Bosworth and Wolters 2001), but we present only lateral measures here because dorsal and lateral measures resulted in similar predictive patterns (S. Pothoven, unpublished data). Triplicate BIA measures were taken on 32 fish ( 3 yellow perch, 11 walleyes, and 18 lake whitefish) to determine variability of BIA measures on individual fish.

Field measures of resistance and reactance are based on measurements of a series circuit (Kotler et al. 1996) and must be converted to parallel measures by using standard equations, such that

$$
X_{p}=X_{m}+\left(R_{m}^{2} / X_{m}\right)
$$

and

$$
R_{p}=R_{m}+\left(X_{m}^{2} / R_{m}\right),
$$

where $X_{p}=$ parallel reactance, $X_{m}=$ series reactance, $R_{p}$ $=$ parallel resistance, and $R_{m}=$ series resistance. As recommended by the BIA instrument manufacturer, we used parallel measures for all BIA predictions of proximate composition, because parallel measurements most closely approximate real electrical properties of biological tissue.

In the laboratory, frozen fish were homogenized in a commercial grinder and blender. To determine dry-towet mass (percent dry mass; Hartman and Brandt 1995), we dried a 20-30-g subsample of ground fish tissue at $70^{\circ} \mathrm{C}$ to a constant mass (about $3 \mathrm{~d}$ ). For energy density ( $\mathrm{J} / \mathrm{g}$ wet mass), the dried material was ground with a mortar and pestle, and a 1-g subsample was combusted in a Parr 1261 isoperibol bomb calorimeter that was standardized with benzoic acid. To estimate within-fish variability for bomb calorimetry, we initially quantified energy density in triplicate for individual fish of each species. Mean coefficients of variation $(\mathrm{CV}=100 \times \mathrm{SD} /$ mean $)$ for individual fish were low: $1.31 \%(n=5)$ for yellow perch, $1.02 \%(n=$ 10) for walleyes, and $1.19 \%(n=6)$ for lake whitefish. Thus, we subsequently quantified energy density in only one subsample per fish.

For lipid analysis, a subsample of the ground homogenate was freeze-dried, reground with a wand blender, sifted through a $1.68-\mathrm{mm}$ sieve, and stored at $-80^{\circ} \mathrm{C}$ until lipid analysis. To extract lipids, triplicate subsamples of 1-3 mg (measured to nearest $0.001 \mathrm{mg}$ ) of finely homogenized freeze-dried samples were weighed directly into glass test tubes $(50 \mathrm{~mm} \times 6$ $\mathrm{mm}$ ) and combined with $200 \mu \mathrm{L}$ of $2: 1$ chloroform:methanol (HPLC-grade). Homogenization was performed promptly on ice, to avoid evaporation. Samples were homogenized for $30 \mathrm{~s}$ by means of a dremel tool with a glass bead pestle. Each homogenate was decanted into a $1.5-\mathrm{mL}$ conical centrifuge tube. A second $200 \mu \mathrm{L}$ of $2: 1$ chloroform:methanol was added to each test tube and homogenized briefly $(\sim 5 \mathrm{~s})$, acting as a rinse. This second homogenate was then combined with the first homogenate, and $200 \mu \mathrm{L}$ of $0.9 \% \mathrm{NaCl}$ was added (and vortex-mixed) to each tube (Folch et al. 1956). Tubes were centrifuged (Eppendorf, Model 5415, Hamburg, Germany) for $15 \mathrm{~s}$ at $8,000 \mathrm{rpm}$, resulting in a phase separation with nonlipid compounds and methanol partitioned into 
TABLE 1.- Mean total length, wet body mass, energy density, and lipid content for individuals used to develop predictive models of energy density and percent lipids. Ranges of values are given in parentheses; $n=$ sample size.

\begin{tabular}{lccccr}
\hline \multicolumn{1}{c}{ Species } & $n$ & Total length $(\mathrm{mm})$ & Mass $(\mathrm{g})$ & Energy density $(\mathrm{J} / \mathrm{g})$ & Lipid content $(\%)$ \\
\hline Yellow perch & 38 & $247(138-358)$ & $191(29-500)$ & $5,728(3,591-7,782)$ & $5.6(2.7-8.7)$ \\
Walleye & 30 & $503(328-639)$ & $1,362(270-2,670)$ & $7,215(5,731-9,299)$ & $10.0(6.0-18.2)$ \\
Lake whitefish & 34 & $502(246-564)$ & $1,136(80-1,910)$ & $6,184(4,550-7,782)$ & $7.3(2.4-14.7)$ \\
\hline
\end{tabular}

the upper aqueous phase, and lipid material in the lower organic phase. A microsampling pipette was used to remove organic-phase lipid extracts for analysis. In 2005, total lipids were determined with both gravimetric and colorimetric techniques as part of a concurrent methodological study that yielded a 0.98 correlation between methods ( $\mathrm{Lu}$ et al., in press). Only the colorimetric method was used in 2006. The gravimetric analyses, which are based on weighing total extracted lipids, were as described by Gardner et al. (1985). The microcolorimetric sulfophosphovanillan (SPV) analysis, which measured the absorbance of the red-purple complex produced from the reaction between double carbon bonds and the phosphovanillin SPV reagent, followed Van Handel's (1985) description. See $\mathrm{Lu}$ et al. (in press) for more details on our lipid analyses. Percent lipids was expressed as percent of wet body mass.

Previous studies predicted the whole-body energy content of fish by using a "conductor volume" method (e.g., length $\left.{ }^{2} / R_{p}\right)$, which treats an individual fish's body as a true cylinder (Hoffer et al. 1969; Bowen et al. 1999; Cox and Hartman 2005). However, given that the body shapes of most fish are not true cylinders, this approach may induce undue error (Kotler et al. 1996).For this reason, we also used a multiple regression approach to both directly and indirectly derive predictive relationships between BIA measures and energy content. To do so, we evaluated four regression models: (1) a "body mass $(M)$ model" that used $M$ alone as a predictor; (2) a "conductor volume model" that used the BIA-derived measure $\mathrm{TL}^{2} / R_{p}$ as a predictor; (3) a "BIA model" with BIA measures as predictors (TL, $R_{p}$, and $X_{p}$ ); and (4) a "BIA + body mass model" with BIA measures (TL, $R_{p}$, and $X_{p}$ ) and $\mathrm{M}$ as predictors.

For the direct approach, each model was used to predict energy density or percent lipids directly. Because BIA is typically used to evaluate total mass of a proximate component, rather than a proportionalbased metric such as energy density or percent lipids, we also used each model to predict total energy, total lipid mass, and total dry mass. For analysis of total energy, total lipid mass, and total dry mass, both the dependent variables and the predictors $\left(M, \mathrm{TL}, R_{p}, X_{p}\right.$, $\mathrm{TL}^{2} / R_{p}$ ) were $\log _{10}$ transformed to linearize the relationships and stabilize the variances. We then indirectly calculated energy density and percent lipids as total energy (or total lipids) divided by measured wet-body mass. For both approaches, we evaluated models for interactions among species and other predictors. When slopes of at least one of the regression models varied among species for a given metric (i.e., species interactions were evident), we made predictions for species-specific relationships.

To select the most parsimonious ("best") models (Spendelow et al. 1995), we used a second-order Akaike information criterion for small sample sizes $\left(\mathrm{AIC}_{c} ; n_{i} / k_{i}<40 ;\right.$ ) to rank models (Burnham and Anderson 1998). We then subtracted the lowest $\mathrm{AIC}_{c i}$ value for the suite of models $\left(\mathrm{AIC}_{c[\mathrm{~min})}\right)$ from each individual $\mathrm{AIC}_{c i}$ value, producing a ranked index referred to as $\Delta_{i}-\mathrm{AIC}_{c(\min )}$; for the most-parsimonious model, $\Delta_{i}-$ AIC $_{c(\min )}=0$.

We used an independent validation group of fish to asses the robustness of both the direct and indirect regression-derived predictions of energy density and percent lipids. This validation set of fish consisted of seven randomly selected fish of each species that were not used to build any of the predictive relationships. To assess whether our direct and indirect predictions of energy density and percent lipids differed from the observed values, we used $95 \%$ confidence intervals to determine whether the slopes of the relationships between predicted and observed values differed from 1 and whether the intercepts differed from 0 . We also quantified the percent error between predicted and observed values for energy density and percent lipids for each fish in the validation group. A two-factor analysis of variance (ANOVA) was then used to examine the effects of species and model on percent error. We used SYSTAT 10 to perform all statistical analyses and evaluated effects at $\alpha=0.05$ level of significance.

\section{Results}

We quantified BIA indices for 123 fish ( 37 walleyes, 45 yellow perch, and 41 lake whitefish) ranging in size from $138 \mathrm{~mm}(29 \mathrm{~g})$ to $639 \mathrm{~mm}(2,670 \mathrm{~g})$ (Table 1). The mean coefficient of variation (based on triplicate measures of individual fish) of lateral BIA measures was lower for $R_{p}(2.89 \%)$ than $X_{p}(8.32 \%)$. There was 
TABLE 2.- Regression models for predicting energy density and percent lipids for yellow perch, walleyes, and lake whitefish. The predictor variables are as follows: body mass $(M)$, total length (TL), parallel resistance $\left(R_{p}\right)$, a nd parallel reactance $\left(X_{p}\right)$. A second-order Akaike criterion for small sample sizes, $\Delta_{i}-\mathrm{AIC}_{c(\min )}$, was used to rank models, the most parsimonious model having a value of 0 (see Methods for details). The most parsimonious models are indicated by bold type.

\begin{tabular}{|c|c|c|c|c|}
\hline Species & Model & $R^{2}$ & $P$-value & $\Delta_{i}-\mathrm{AIC}_{c(\min )}$ \\
\hline \multicolumn{5}{|c|}{ Energy density } \\
\hline \multirow[t]{4}{*}{ Yellow perch } & $6153-2.23(M)$ & 0.06 & 0.13 & 0.410 \\
\hline & $5998-1.38\left(\mathrm{TL}^{2} / R_{p}\right)$ & 0.02 & 0.37 & 1.994 \\
\hline & $9247-8.15(\mathrm{TL})-4.33\left(R_{p}\right)-0.03\left(X_{p}\right)$ & 0.18 & 0.08 & 0.000 \\
\hline & $8635-3.55(\mathrm{TL})-4.59\left(R_{p}^{p^{\prime}}\right)-0.04\left(X_{p}^{p^{\prime}}-2.22(M)\right.$ & 0.18 & 0.15 & 2.321 \\
\hline \multirow[t]{4}{*}{ Walleye } & $6264+0.70(M)$ & 0.38 & $<0.001$ & 6.284 \\
\hline & $5924+1.47\left(\mathrm{TL}^{2} / R_{n}\right)$ & 0.50 & $<\mathbf{0 . 0 0 1}$ & 0.000 \\
\hline & $8441-2.15(\mathrm{TL})-8.71\left(R_{p}\right)+0.51\left(X_{p}\right)$ & 0.49 & $<0.001$ & 5.357 \\
\hline & $11436+6.85(\mathrm{TL})-10.08^{p}\left(R_{n}\right)+0.84^{p}\left(X_{n}\right)+1.21(M)$ & 0.56 & $<0.001$ & 3.642 \\
\hline \multirow[t]{4}{*}{ Lake whitefish } & $4671+1.33(M)$ & 0.29 & 0.001 & 0.000 \\
\hline & $5236+1.02\left(\mathrm{TL}^{2} / R_{n}\right)$ & 0.09 & 0.08 & 8.251 \\
\hline & $4416+4.39(\mathrm{TL})-4.93\left(R_{p}\right)+1.50\left(X_{p}\right)$ & 0.29 & 0.02 & 4.675 \\
\hline & $6324-3.81(\mathrm{TL})-2.71\left(R_{p}^{p}\right)+0.93\left(X_{p}^{p^{\prime}}\right)+1.71(M)$ & 0.37 & 0.01 & 3.190 \\
\hline \multicolumn{5}{|c|}{$\%$ Lipids } \\
\hline \multirow[t]{4}{*}{ Yellow perch } & $6.1-0.003(M)$ & 0.03 & 0.33 & 1.600 \\
\hline & $5.9-0.001\left(\mathrm{TL}^{2} / R_{n}\right)$ & 0.01 & 0.69 & 2.431 \\
\hline & $11.8-0.012(\mathrm{TL})-0.008\left(R_{n}\right)-0.003\left(X_{n}\right)$ & 0.17 & 0.09 & 0.000 \\
\hline & $11.0-0.007(\mathrm{TL})-0.008\left(R_{p}^{p^{\prime}}\right)-0.001\left(X_{p}\right)^{\prime}+0.003(M)$ & 0.18 & 0.16 & 2.407 \\
\hline \multirow[t]{4}{*}{ Walleye } & $7.7+0.002(M)$ & 0.18 & 0.02 & 2.756 \\
\hline & $6.8+0.004\left(\mathrm{TL}^{2} / R_{p}\right)$ & 0.25 & 0.01 & 0.000 \\
\hline & $7.1+0.012(\mathrm{TL})+0.002\left(R_{p}\right)-0.004\left(X_{p}\right)$ & 0.28 & 0.03 & 3.515 \\
\hline & $13.6-0.008(\mathrm{TL})-0.001\left(R_{p}\right)-0.003\left(X_{p}\right)+0.003(M)$ & 0.31 & 0.05 & 5.011 \\
\hline \multirow[t]{4}{*}{ Lake whitefish } & $1.6+0.005(M)$ & 0.36 & $<0.001$ & 3.246 \\
\hline & $4.0+0.004\left(\mathrm{TL}^{2} / R_{n}\right)$ & 0.10 & 0.07 & 14.807 \\
\hline & $1.3+0.015(\mathrm{TL})-0.021\left(R_{p}\right)+0.007\left(X_{p}\right)$ & 0.41 & 0.001 & 4.811 \\
\hline & $8.9-0.018(\mathrm{TL})-0.012\left(R_{p}\right)+0.004\left(X_{p}\right)+0.007(M)$ & 0.53 & $<\mathbf{0 . 0 0 1}$ & 0.000 \\
\hline
\end{tabular}

no indication that the coefficient of variation of BIA measures increased with fish size $\left(R^{2}<0.07\right)$ or varied by species. Although energy density was strongly correlated with percent dry weight (yellow perch: $R^{2}=$ 0.73; walleyes: $R^{2}=0.94$; lake whitefish: $R^{2}=0.90$; all $P<0.001$ ), the relationships between percent lipids and percent dry weight were weaker (yellow perch: $R^{2}$ $=0.47$; walleyes: $R^{2}=0.52$; lake whitefish: $R^{2}=0.51$; all $P<0.001)$.

\section{Direct Approach}

Predictive models were determined separately for each species, owing to significant interactions among species and some predictor variables in the models. For directly predicting energy density, AIC values indicated a different best model for each species: the BIA model for yellow perch, the conductor volume model for walleyes, and the body mass model for lake whitefish (Table 2). However, even the best model fits were far from perfect for walleyes $\left(R^{2}=0.50 ; P<\right.$ $0.001)$ and whitefish $\left(R^{2}=0.29 ; P=0.001\right)$ and were not significant for yellow perch $\left(R^{2}=0.18 ; P=0.08\right)$.

As with energy density, the variation in percent lipids explained by our best models was imperfect (maximum $R^{2}, 0.53$ ). The best predictive models were the BIA model for yellow perch $\left(R^{2}=0.17 ; P=0.09\right)$, the conductor volume model for walleyes $\left(R^{2}=0.25 ; P\right.$ $<0.01)$, and BIA $+M$ model for lake whitefish $\left(R^{2}=\right.$ $0.53 ; P<0.001)$.

\section{Indirect Approach}

The body mass model (for yellow perch) and the BIA $+M$ model (for lake whitefish) were identified as the most-parsimonious models for predicting $\log _{10}$ transformed total energy, total lipid mass, and dry body mass (Table 3). For walleyes, the body mass model was the best at predicting total lipids, but the BIA $+M$ model was the best at predicting total energy and dry body mass (Table 3). All of our most-parsimonious models explained the majority of variation in total energy, total lipid mass, and dry mass (all $R^{2} \geq 0.76$; all $P<0.001$ ).

\section{Model Validation}

When used to predict total energy, total lipid mass, or dry mass for the validation group of fish, the BIA + $M$ equations had the strongest correlation between observed and predicted values, although these correlations were only slightly better than those for the body mass models (Figure 1). All relationships between observed and predicted values were significant $(P<$ 0.001), and in all cases the slopes and intercepts did not differ from 1 and 0 , respectively. 
TABLE 3.-Coefficents of determination $\left(R^{2}\right)$ and Akaike rankings (in parentheses) for four sets of regression models used to predict total energy $(J)$, total lipid mass $(\mathrm{g})$, and total dry mass $(\mathrm{g})$ for yellow perch, walleyes, and lake whitefish. All models were significant $(P<0.001)$. Predictor variables (see Table 2 ) were $\log _{10}$ transformed. A second-order Akaike criterion for small sample sizes was used to rank models by relative parsimony; the most parsimonious models are indicated by bold type.

\begin{tabular}{|c|c|c|c|c|c|}
\hline \multirow[b]{2}{*}{ Species } & \multirow[b]{2}{*}{ Metric } & \multicolumn{4}{|c|}{ Model } \\
\hline & & $a+b_{1}(M)$ & $a+b_{1}\left(\mathrm{TL}^{2} / R_{p}\right)$ & $\begin{array}{c}a+b_{0}(\mathrm{TL}) \\
+b_{1}\left(R_{p}\right)+b_{2}\left(X_{p}\right) \\
\end{array}$ & $\begin{array}{l}a+b_{0}(\mathrm{TL})+b_{1}\left(R_{p}\right) \\
\quad+b_{2}\left(X_{p}\right)+b_{3}(M) \\
\end{array}$ \\
\hline \multirow[t]{3}{*}{ Yellow perch } & Energy & $0.927(0.000)$ & 0.848 (27.793) & $0.906(13.943)$ & $0.934(3.290)$ \\
\hline & Lipids & $0.763(0.000)$ & $0.747(2.517)$ & $0.777(2.325)$ & $0.799(0.945)$ \\
\hline & Dry mass & $0.974(0.000)$ & 0.887 (55.289) & $0.956(23.444)$ & $0.977(1.481)$ \\
\hline \multirow[t]{3}{*}{ Walleye } & Energy & $0.987(4.011)$ & $0.930(53.621)$ & $0.974(29.037)$ & $0.991(0.000)$ \\
\hline & Lipids & $0.920(0.000)$ & $0.882(11.608)$ & $0.910(8.398)$ & $0.932(2.547)$ \\
\hline & Dry mass & $0.995(5.268)$ & $0.930(84.545)$ & $0.983(47.312)$ & $0.997(0.000)$ \\
\hline \multirow[t]{3}{*}{ Lake whitefish } & Energy & $0.960(2.854)$ & $0.748(65.678)$ & $0.912(34.462)$ & $0.971(0.000)$ \\
\hline & Lipids & $0.838(1.489)$ & $0.621(30.426)$ & $0.796(14.166)$ & $0.875(0.000)$ \\
\hline & Dry mass & $0.980(7.789)$ & $0.756(93.611)$ & $0.933(54.456)$ & $0.987(0.000)$ \\
\hline
\end{tabular}

When used to predict energy density for the validation group of fish, the BIA $+M$ equations had the strongest correlation between observed and predicted values for both the direct and indirect approaches (Figure 2). All relationships between observed and predicted values were significant $(P<0.03)$, except for conductor volume indirect equations $(P=0.12)$. In all cases, the slope was significantly less than 1 and the intercept was significantly greater than 0 , indicating that our models tended to underestimate energy density for individuals with a high energy density and overestimate energy density for individuals with a low energy density.

The correlations between the predicted and observed values of percent lipids were weaker than the
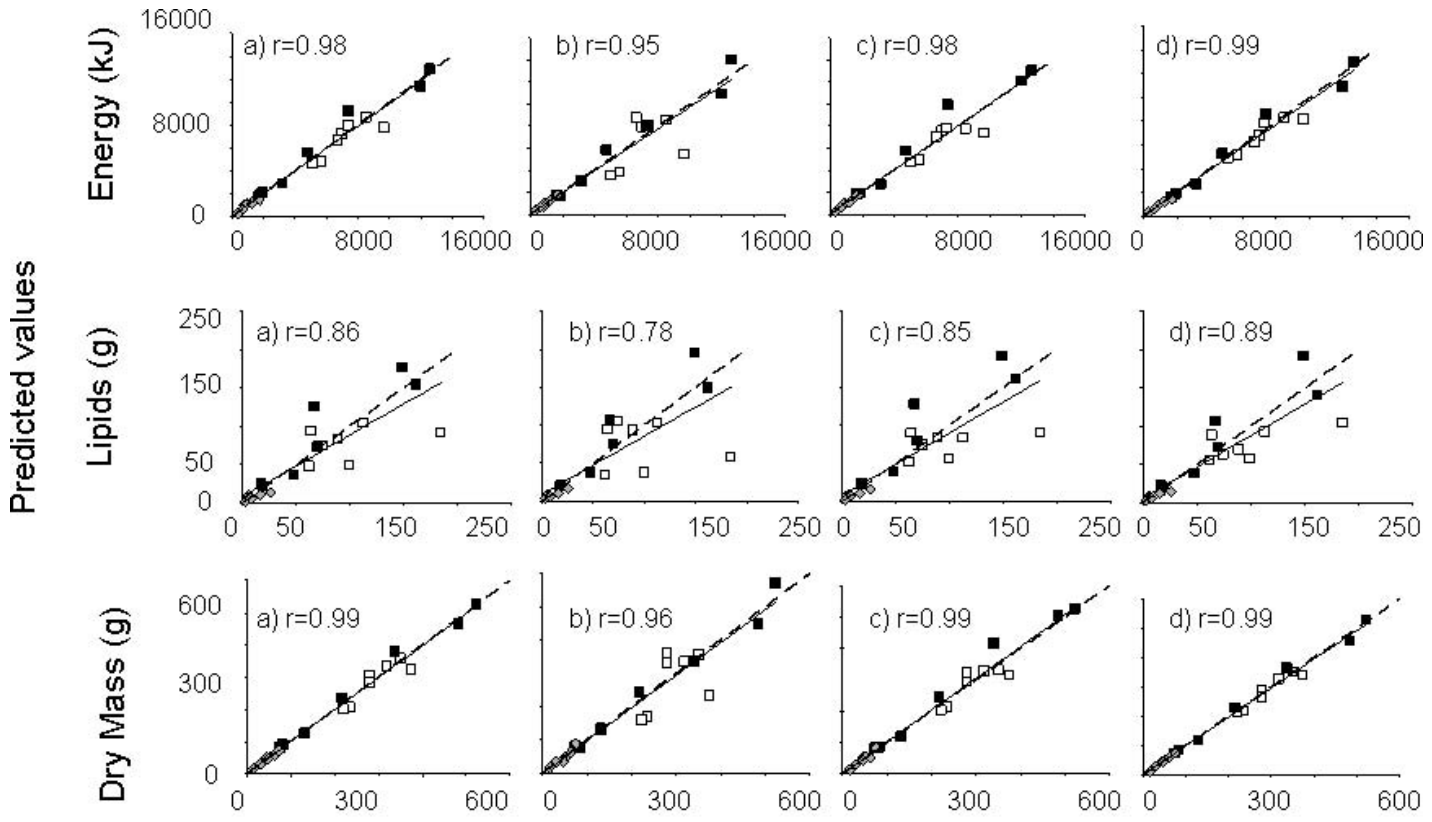

\section{Observed values}

FIGURE 1.-Observed versus predicted values of total energy, total lipid mass, and total dry mass using species-specific regression models with the predictors (a) body mass, (b) conductor volume, (c) bioelectrical impedance analysis (BIA) measures only, and (d) BIA measures combined with body mass for a validation group of fish ( $n=7$ individuals/species). See text for details on models. The solid line represents the regression line, the dashed line a 1:1 relationship between observed and predicted values. Diamonds represent values for yellow perch, closed squares values for walleyes, and open squares values for lake whitefish. 

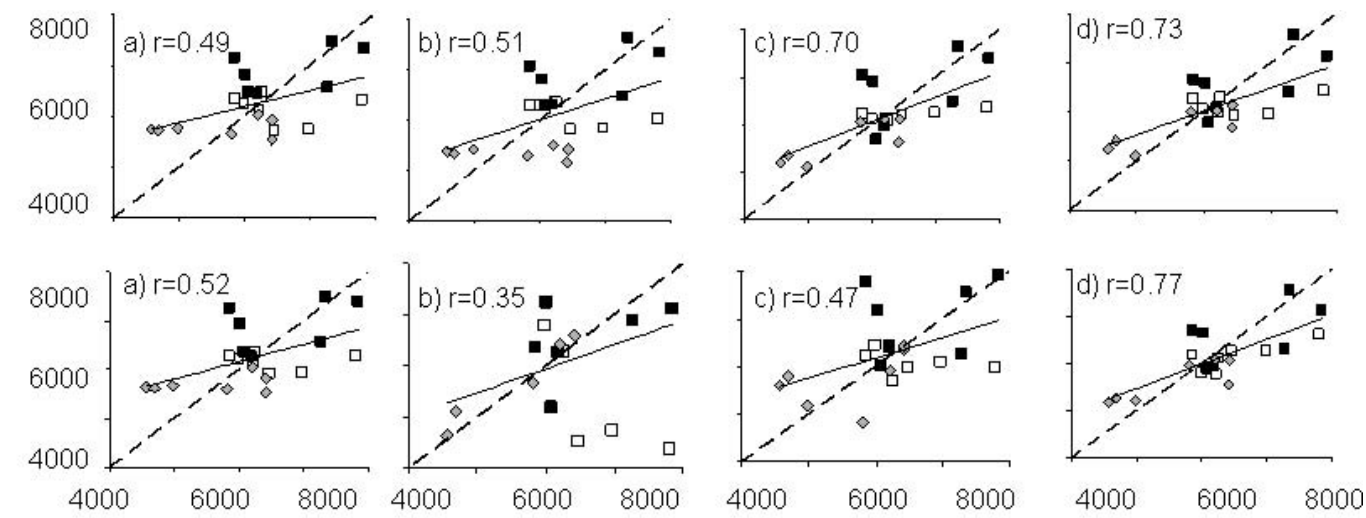

\section{Observed energy density $(\mathrm{J} / \mathrm{g})$}

FIGURE 2.- - Observed versus predicted values of energy density derived directly (top row) and indirectly (bottom row) using species-specific regression models with the predictors (a) body mass, (b) conductor volume, (c) bioelectrical impedance analysis (BIA) measures only, and (d) BIA measures combined with body mass for a validation group of fish ( $n=7$ individuals/species). See Figure 1 for additional details.

analogous comparisons for energy density (Figure 3). The relationship between observed and predicted values was significant for only the BIA $+M$ direct and indirect models $(P<0.01)$. For these models, the slope was again significantly less than 1 , and the intercept was significantly greater than 0 .

There was no difference in the percent error between observed and predicted values for either energy density or percent lipids across models $(P>0.25$; Table 4$)$ or species $(P>0.05)$. The maximum percent error between the predicted and observed values of energy density was lowest (15-18\%) for models that combined
BIA measures with fish mass. For percent lipids, the lowest maximum percent errors were for models that used some type of BIA measure, but were still extremely high (82-89\%).

\section{Discussion}

Our analyses demonstrate that models containing BIA measures provide solid predictions of total dry mass, total energy, and total lipids for our three study species, which corresponds to Cox and Hartman's (2005) results from a laboratory study conducted with brook trout Salvelinus fontinalis. In that study, Cox and
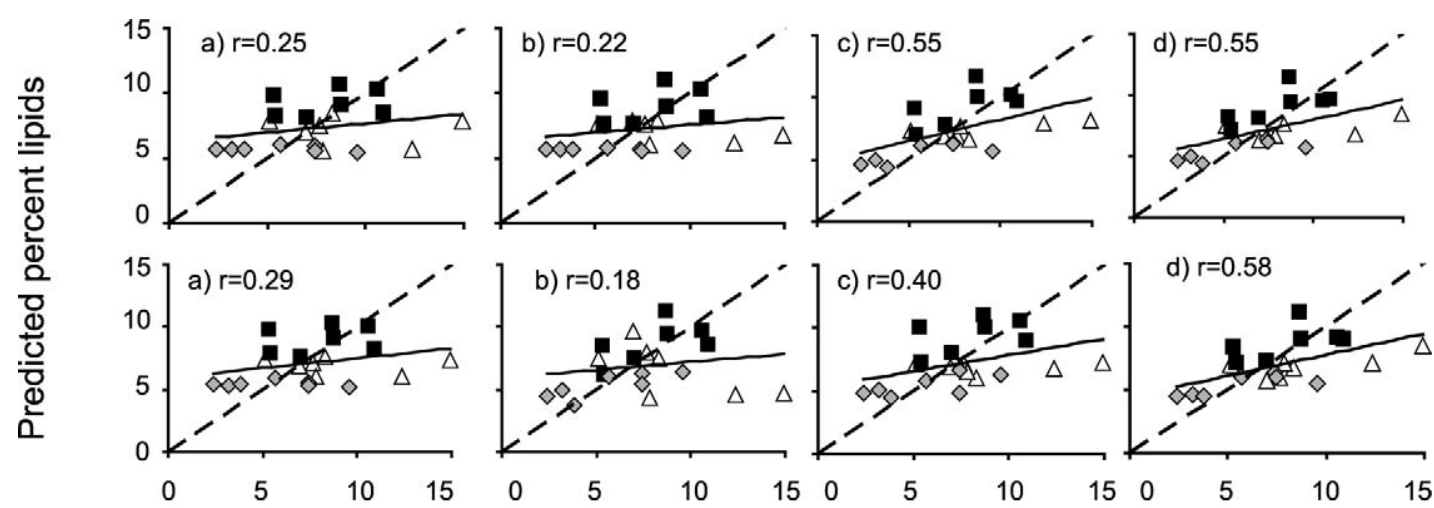

Observed percent lipids

FIGURE 3.-Observed versus predicted values of percent lipids derived directly (top row) and indirectly (bottom row) by using species-specific regression models with the predictors (a) body mass, (b) conductor volume, (c) bioelectrical impedance analysis (BIA) measures only, and (d) BIA measures combined with body mass for a validation group of fish ( $n=7$ individuals/species). Diamonds represent values for yellow perch, squares values for walleyes, and triangles values for lake whitefish. See Figure 1 for additional details. 
TABLE 4.-Average percent differences (ranges in parentheses) between predicted and observed values of energy density and percent lipids for the validation group of fish (7 yellow perch, 7 walleyes, and 7 lake whitefish) with four different regression models, where energy density and percent lipids are calculated both directly and indirectly (see text). Predictor variables are defined in Table 2.

\begin{tabular}{llllrr}
\hline \multirow{2}{*}{ Metric } & & \multicolumn{4}{c}{ Model } \\
\cline { 3 - 5 } & Calculation & $a+b_{1}(M)$ & $a+b_{1}\left(\mathrm{TL}^{2} / R_{p}\right)$ & $\begin{array}{c}a+b_{0}(\mathrm{TL}) \\
+b_{1}\left(R_{p}\right)+b_{2}\left(X_{p}\right)\end{array}$ & $\begin{array}{c}a+b_{0}(\mathrm{TL})+b_{1}\left(R_{p}\right) \\
+b_{2}\left(X_{p}\right)+b_{3}(M)\end{array}$ \\
\hline \multirow{2}{*}{ Energy density } & Direct & $10(2-26)$ & $10(1-23)$ & $8(1-20)$ & $8(1-18)$ \\
& Indirect & $10(1-24)$ & $13(1-44)$ & $10(0-33)$ & $8(2-15)$ \\
& Direct & $36(1-134)$ & $35(0-134)$ & $27(2-89)$ & $28(6-97)$ \\
& Indirect & $33(1-123)$ & $31(1-82)$ & $30(0-97)$ & $27(3-83)$ \\
\hline
\end{tabular}

Hartman found that predictive relationships with BIA measures could explain more than $95 \%$ of the variation in dry body mass, total lipid content, and total water content. Although the point is not discussed in Cox and Hartman (2005), our results also show that the addition of BIA measures to models with body mass alone slightly improves the predictive capabilities of those models. In fact, for all three species, the increase in the amount of variation explained by the addition of BIA measures was less than $5 \%$ for all body constituents. Further, in the case of yellow perch, we found that models with BIA measures were not any better than models with only body mass for predicting total energy, lipid mass, and dry mass.

Evaluations of BIA with humans (Mazess 1998) and small mammals (Wirsing et al. 2002) have also indicated that body mass can be as good a predictor of total-body water content as BIA measures. Lantry et al. (1999) drew a similar conclusion using TOBEC to estimate fish total-body water. This suite of results, including our study, is not surprising, given that animal mass alone can explain over $90 \%$ of the variation in total mass of proximate body measures for many organisms (see Bosworth and Wolters 2001). Additionally, the strong ability of models with BIA measures to accurately predict the total mass of body components may simply be related to the adjustment of BIA measures by length or length squared (Forbes et al. 1992; Mazess 1998), although others have argued that the physical properties of electricity passing through tissues necessitates such length-based adjustments to BIA measures (Hoffer et al. 1969; Kushner et al. 1992; Bracco et al. 1996).

The predictions of energy density and percent lipids from BIA measures were much less reliable than those for total energy, lipids, or dry mass. For the direct approach, regression models that contained BIA measures (BIA model, conductor volume model, and BIA + body mass model) were generally the best (most-parsimonious) models for estimating energy density or percent lipids; however, even these models explained at most $53 \%$ of the variation in observed energy density or percent lipids, and for yellow perch, none of the models were even significant. Our findings support Bosworth and Wolters (2001), who hypothesized that the correlations between BIA measures and total water or fat would be substantially lower when these proximate components were expressed as percentages.

For both the indirect and direct approaches, models that combined BIA measures with body mass had the strongest correlations between the predicted and observed values of energy density $(r=0.73-0.77)$ and percent lipids $(r=0.55-0.58)$. There were significant biases in these predictions, however. In particular, these models underestimated energy density and percent lipids for energetically rich individuals and overestimated energy density and percent lipids for individuals with fewer energy reserves. Although the maximum percent error between predicted and observed values was lowest for models that incorporated BIA measures, these errors were still rather high for both energy density (15-18\%) and percent lipids (82$89 \%$ ). Similarly, Bowen et al. (1999) concluded that, even though BIA was found to be a decent predictor for gray seal total-body water, individual estimates were often associated with a high degree of error (up to $25 \%$ ). Further, a study with moose indicated that BIA did not provide precise predictions of body fat and concluded that the method was unsuitable for use in the field (Hundertmark and Schwartz 2002).

In contrast to our findings, Bosworth and Wolters (2001) found that including BIA measures in models with fish mass improved predictions over those that used body mass alone for both percent moisture $\left(R^{2}=\right.$ 0.65 versus 0.25$)$ and percent fat $\left(R^{2}=0.75\right.$ versus 0.04 ) of channel catfish Ictuluras punctatus. In that study, however, only deheaded, eviscerated, and skinned carcasses were used for laboratory determinations of body moisture and fat (Bosworth and Wolters 2001). In our study, as in two previous studies with fish (Bosworth and Wolters 2001; Cox and Hartman 2005), 
BIA measures were taken to encompass the body of a fish between the posterior edge of the operculum and the caudal peduncle region. Thus, the head of the fish was not included in the area measured between the two sets of electrodes. It was, however, included in determinations of whole-body energy density or percent lipids because we homogenized the entire fish. Given the large amount of bony material in the head region, it is probably a low-energy region, and when combined with the rest of the body, could lower the overall percent energy content. However, the bony head region also may have high resistance, similar to that of lipids, which could further bias BIA readings. We recommend that future studies vary the placement of electrodes to account for whole-body (including head) resistance and reactance to try and improve BIA predictions of whole-fish energy content on a percentage basis.

Other factors may also contribute to the poor ability of BIA to predict energy density and percent lipids. For humans, the various demographics or body builds of people are linked to variation in predictive equations (Kotler et al. 1996; Chumlea et al. 2002; Kyle et al. 2004), so similar difficulties may occur when studying various species of fish from different environmental conditions or locations. Also, where lipids are stored within a fish can change ontogenetically or seasonally without affecting overall energy density, and such alterations could influence BIA measures and predictions. For example, fish may mobilize somatic and visceral lipids for gonadal development without altering their overall energy content (Henderson et al. 1996). Cox and Hartman (2005) suggested that fat in the ventral regions may not be well represented by lateral BIA measures. In turn, alterations in ventral fat deposits that do not correspond with any overall change in energy density could readily bias BIA predictions. Finally, we did not account for variation in ambient air or body temperature, which may influence BIA readings (Buono et al. 2004).

In contrast to BIA, a handheld microwave energy meter (fat meter), which requires species-specific regressions of meter readings versus proximate components, has provided strong predictive power $\left(R^{2}>\right.$ 0.93 ) for the somatic percent lipids and energy densities of salmonids (Crossin and Hinch 2005); however, the relationships are weaker for spawning fish $\left(R^{2}=0.49-0.87\right.$; Hendry and Beall 2004). The fat meter measures water content and estimates lipid content using published water-to-lipid relationships, which can vary across species (Crossin and Hinch 2005) and even within a species (Pothoven et al. 2006). Thus, some evaluation would be necessary before this method is applied generally (Hendry and Beall 2004).
Additionally, this technique does not account for viscera and has been restricted to analyzing somatic tissues. As such, we are uncertain as to whether this method can reliably estimate whole-body energy content, which is necessary for bioenergetics applications.

Clearly, BIA techniques are not currently reliable enough to allow for routine determinations of wholefish energy density or percent lipids. The added value of the BIA metrics over simply weighing individuals was marginal, and significant biases were found for predictions relative to observed values. However, difficulties in applying relatively new technology to a problem should not come as a surprise. BIA techniques have been used with humans since the 1970s, and the methods are still being refined and debated (Kushner et al. 1992; Mazess 1998; Kyle et al. 2004). During the development of BIA techniques for humans, Jackson et al. (1988) indicated that validation of the technique would require multiple investigators sampling large, heterogeneous samples. Similar challenges and requirements should be expected if BIA is further pursued as a routine research technique for fisheries sciences.

\section{Acknowledgments}

Thanks to those who provided assistance in the field and laboratory, including J. Breck, E. Rutherford, J. Roberts, S. Sisler, C. Hand, M. Constantini, the crew of RV Laurentian, B. Sylak, J. Cudney, and A. GaschoLandis, and the Ohio Department of Natural Resources-Division of Wildlife. This research was supported by the National Oceanic and Atmospheric Administration, Great Lakes Environmental Research Laboratory (NOAA-GLERL) and is NOAA-GLERL contribution 1470. Mention of a commercial company or product does not constitute an endorsement by NOAA.

\section{References}

Bartell, S. M., J. E. Breck, R. H. Gardner, and A. L. Brenkert. 1986. Individual parameter perturbation and error analysis of fish bioenergetics models. Canadian Journal of Fisheries and Aquatic Sciences 43:160-168.

Bosworth, B. G., and W. R. Wolters. 2001. Evaluation of bioelectrical impedance to predict carcass yield, carcass composition, and fillet composition in farm-raised catfish. Journal of the World Aquaculture Society 32:72-78.

Bowen, W. D., C. A. Beck, and S. J. Iverson. 1999. Bioelectrical impedance analysis as a means of estimating total body water in grey seals. Canadian Journal of Zoology 77:418-422.

Bracco, D., D. Thiebaud, R. L. Chiolero, M. Landry, P. Burkhardt, and Y. Schutz. 1996. Segmental body composition assessed by bioelectrical impedance analysis 
and DEXA in humans. Journal of Applied Physiology 81:2580-2587.

Buono, M. J., S. Burke, S. Endemann, H. Graham, C. Gressard, L. Griswold, and B. Michalewicz. 2004. The effect of ambient air temperature on whole-body bioelectrical impedance. Physiological Measurement 25:119-123.

Burnham, K. P., and D. R. Anderson. 1998. Model selection and inference: a practical information-theoretic approach. Springer-Verlag, New York.

Chumlea, W. C., S. S. Guo, R. J. Kuczmarski, K. M. Flegal, C. L. Johnson, S. B. Heymsfield, H. C. Lukaski, K. Friedl, and V. S. Hubbard. 2002. Body composition estimates from NHANES III bioelectrical impedance data. International Journal of Obesity 26:1596-1609.

Cox, M. K., and K. J. Hartman. 2005. Nonlethal estimation of proximate composition in fish. Canadian Journal of Fisheries and Aquatic Sciences 62:269-275.

Crossin, G. T., and S. G. Hinch. 2005. A nonlethal, rapid method for assessing the somatic energy content of migrating adult Pacific salmon. Transactions of the American Fisheries Society 134:184-191.

Folch, J., M. Lees, and G. H. Sloane Stanley. 1956. A simple method for the isolation and purification of total lipids from animal tissues. Journal of Biological Chemistry 226:497-509.

Forbes, G. B., W. Simon, and J. M. Amatruda. 1992. Is bioimpedance a good predictor of body composition change? American Journal of Clinical Nutrition 56:4-6.

Gardner, W. S., W. A. Frez, E. A. Cichocki, and C. C. Parrish. 1985. Micromethod for lipids in aquatic invertebrates. Limnology and Oceanography 30:1099-1105.

Hanson, P. C., T. B. Johnson, D. E. Schindler, and J. F. Kitchell. 1997. Fish bioenergetics 3.0. University of Wisconsin, Sea Grant Institute, Technical Report WISCU-T-97-001, Madison.

Hartman, K. J., and S. B. Brandt. 1995. Estimating energy density of fish. Transactions of the American Fisheries Society 124:347-355.

Henderson, B. A., J. L. Wong, and S. J. Nepszy. 1996. Reproduction of walleye in Lake Erie: allocation of energy. Canadian Journal of Fisheries and Aquatic Sciences 53:127-133.

Hendry, A. P., and E. Beall. 2004. Energy use in spawning Atlantic salmon. Ecology of Freshwater Fish 13:185196.

Hoffer, E. C., C. K. Meador, and D. C. Simpson. 1969 Correlation of whole-body impedance with total body water volume. Journal of Applied Physiology 27:531534.

Hundertmark, K. J., and C. C. Schwartz. 2002. Evaluation of bioelectrical impedance analysis as an estimator of moose body composition. Wildlife Society Bulletin 30:1-7.

Hurst, T. P., and D. O. Conover. 2003. Seasonal and interannual variation in the allometry of energy allocation in juvenile striped bass. Ecology 84:3360-3369.

Jackson, A. S., M. L. Pollock, J. E. Graves, and M. T. Mahar. 1988. Reliability and validity of bioelectrical impedance in determining body composition. Journal of Applied Physiology 64:529-534.

Kotler, D. P., S. Burastero, J. Wang, and R. N. Pierson, Jr.
1996. Prediction of body cell mass, fat-free mass, and total body water with bioelectrical impedance analysis: effects of race, sex, and disease. American Journal of Clinical Nutrition 64:489S-497S

Kushner, R. F., D. A. Schoeller, C. R. Fjeld, and L. Danford. 1992. Is the impedance index $\left(\mathrm{ht}^{2} / R\right)$ significant in predicting total body water? American Journal of Clinical Nutrition 56:835-839.

Kyle, U. G., I. Bosaeus, A. D. De Lorenzo, P. Deurenberg, M. Elia, J. M. Gomez, B. L. Heitmann, L. Kent-Smith, J. Melichor, M. Pirlich, H. Scharfetter, A. M. W. J. Schols, and C. Pichard. 2004. Bioelectrical impedance analysis, part 1: review of principles and methods. Clinical Nutrition 23:1226-1243.

Lantry, B. F., D. J. Stewart, P. S. Rand, and E. L. Mills. 1999. Evaluation of total-body electrical conductivity to estimate whole-body water content of yellow perch, Perca flavescens, and alewife, Alosa pseudoharengus. U.S. National Marine Fisheries Service Fishery Bulletin 97:71-79.

Lu, Y., S. A. Ludsin, D. L. Fanslow, and S. A. Pothoven. In press. Comparison of three micro-quantity techniques for measuring total lipids in fish. Canadian Journal of Fisheries and Aquatic Sciences.

Ludsin, S. A., and D. R. DeVries. 1997. First-year recruitment of largemouth bass: the interdependency of early life stages. Ecological Applications 7:1024-1038.

Lukaski, H. C., P. E. Johnson, W. W. Bolonchik, and G. I. Lykken. 1985. Assessment of fat-free mass using bioelectrical impedance measurements of the human body. American Journal of Clinical Nutrition 41:810 817.

Madenjian, C. P., R. F. Elliot, T. J. DeSorcie, R. M. Stedman, D. V. O'Connor, and D. V. Rottiers. 2000. Lipid concentrations in Lake Michigan fishes: seasonal, spatial, ontogenetic, and long-term trends. Journal of Great Lakes Research 26:427-444.

Madenjian, C. P., S. A. Pothoven, J. M. Dettmers, and J. D. Holuszko. 2006. Changes in seasonal energy dynamics of alewife (Alosa pseudoharengus) in Lake Michigan after invasion of dreissenid mussels. Canadian Journal of Fisheries and Aquatic Sciences 63:891-902.

Mazess, R. B. 1998. Does bioelectrical impedance analysis predict body composition independently of anthropometry? Journal of Applied Physiology 84:396.

Paine, R. T. 1971. The measurement and application of the calorie to ecological problems. Annual Review of Ecology and Systematics 2:145-164.

Pothoven, S. A., T. F. Nalepa, C. P. Madenjian, R. R. Rediske, P. J. Schneeberger, and J. X. He. 2006. Energy density of lake whitefish Coregonus clupeaformis in Lakes Huron and Michigan. Environmental Biology of Fishes 76:151158.

Rottiers, D. V., and R. M. Tucker. 1982. Proximate composition and caloric content of eight Lake Michigan fishes. U.S. Fish and Wildlife Service Technical Papers 108.

Shearer, K. D. 1994. Factors affecting the proximate composition of cultured fishes with emphasis on salmonids. Aquaculture 119:63-88.

Spendelow, J. A., J. D. Nichols, I. C. T. Nisbet, H. Hays, G. D. Cormons, J. Burger, C. Safina, J. E. Hines, and M. 
Gochfield. 1995. Estimating annual survival and movement rates of adults within a metapopulation of roseate terns. Ecology 76:2415-2428.

Van Handel, E. 1985. Rapid determination of total lipids in mosquitoes. Journal of the American Mosquito Control Association 1:302-304.

Vondracek, B., B. D. Giese, and M. G. Henry. 1996. Energy density of three fishes from Minnesota waters of Lake Superior. Journal of Great Lakes Research 22:757-764.

Wirsing, A. J., T. D. Steury, and D. L. Murray. 2002. Noninvasive estimation of body composition in small mammals: a comparison of conductive and morphometric techniques. Physiological and Biochemical Zoology 75:489-497. 